\title{
ANATOMÍA FOLIAR Y DEL PECÍOLO DE ESPECIES DEL GÉNERO RHUS S. STR. (ANACARDIACEAE)
}

\author{
Agustina Rosa Andrés-HeRnÁndeZ Y y Teresa TeRRAZAS² \\ 'Programa de Botánica, Colegio de Postgraduados, Montecillo, Edo. de México, \\ C.P. 56230, México. Correo-e: arah@colpos.mx \\ 2Departamento de Botánica, Instituto de Biología, Universidad Nacional Autónoma de México, Apdo. Postal 70-233, \\ México 04510, D.F., México. Correo-e: tterrazas@ibiologia.unam.mx
}

\begin{abstract}
Resumen: Se describe la anatomía foliar y del pecíolo de 33 especies de Rhus s. str. con el objetivo de evaluar los atributos anatómicos que apoyen su clasificación infragenérica. Los resultados apoyan la clasificación infragenérica existente de Rhus $s$. str. El subgénero Rhus, con sistema vascular tipo I y pecíolo con más de diez capas de colénquima y médula amplia, se distingue del subgénero Lobadium. La sección Terebinthifolia se caracteriza por tener dos o cuatro elementos de vaso, vaina colenqu im at o s a rodeando los haces vasculares y fibras gelatinosas; mientras que la secciónStyphonia p resenta una cutícula gruesa (> $6 \mu \mathrm{m})$, células epidérmicas papilosas, dos capas de parénquima en empalizada y esclerénquima en xilema y floema. La presencia de esclerénquima en la sección Styphonia se correlaciona con la persistencia de las hojas. Seguramente algunos de los atributos foliares mencionados se originaron durante los procesos de radiación del género Rhus s. str. que tuvieron lugar en México.
\end{abstract}

Palabras clave: esclerénquima, Lobadium, Rhus, Styphonia, Terebinthifolia.

\begin{abstract}
Foliar and petiole anatomy of 33 species of Rhus s. str. is described with the main objective of evaluating anatomical traits to support its infrageneric classification. The results support the existing Rhus s. str. infrageneric classification. The subgenus Rhus, with a vascular system type I and more than ten layers of collenchyma and wide pith in the petiole may be readily separated from subgenus Lobadium. In turn, section Terebinthifolia shows two to four vessel elements, collenchymatic sheath surrounding the vascular bundles and gelatinous fibers, while section Styphonia presents a thick cuticle (6 $\mu \mathrm{m})$, papillose epidermal cells, two layers of palisade parenchyma and sclerenchyma in xylem and phloem. The occurrence of sclerenchyma in section Styphonia is correlated with the persistent character of its leaves. Some of the foliar traits mentioned are undoubtedly related with the radiation events of Rhus s. str. that took place in Mexico.
\end{abstract}

Key words: Lobadium, Rhus, sclerenchyma, Styphonia, Terebinthifolia.

$\mathbf{E}$ género Rhus s. str. (Barkley, 1937) incluye alrededor de 35 especies que se caracterizan por las drupas rojas con tricomas glandulares rojos en su superficie (Barkley, 1937; Young, 1975, 1978) y se subdivide en dos subgéneros: Rhus subgen. Rhus y Rhus subgen. Lobadium (Young, 1975, 1978; Miller et al., 2001), ambos presentes en Laurasia (figura 1). El subgénero Rhus comprende diez especies que se distribuyen cuatro en el este de Asia, cuatro en Norteamérica, una especie en el sureste europeo y otra en Hawaii. Las especies de este subgénero se caracterizan por tener las hojas deciduas, imparipinnado compuestas; las flores aparecen después de las hojas y están arregladas en tirsos terminales; cada flor está sostenida por una bráctea también decidua y linear-lanceolada (Barkley, 1937; Young, 1975).

El subgénero Lobadium contiene alrededor de 25 especies agrupadas en tres secciones, Lobadium (5), Styphonia ( 13) y Terebinthifolia (7), las cuales se distribuyen principalmente en el suroeste de los Estados Unidos y México. Este subgénero se caracteriza por presentar las hojas perennes o deciduas, simples, trifolioladas o imparipinnado compuestas; las flores aparecen con o antes de las hojas, en su mayoría son sésiles y se arreglan en espigas compuestas con una bráctea persistente, deltoide u ovada y dos bractéolas que sostienen a cada flor (Young, 1978). 


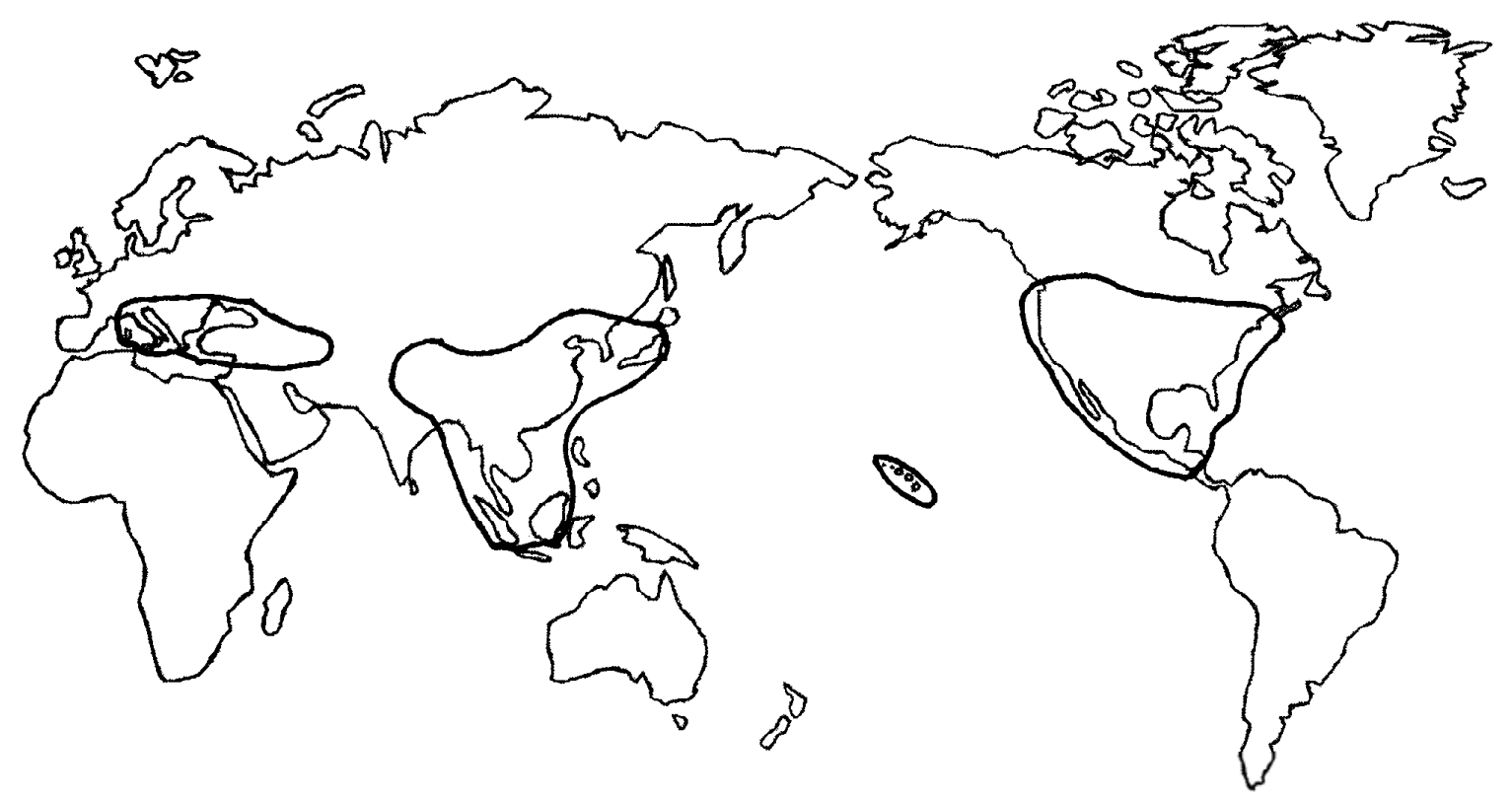

Figura 1. Mapa de distribución del género Rhus s. str.

Los estudios morfológicos y anatómicos para las especies del género Rhus s. str. son escasos (Metcalfe y Chalk, 1950). Por ejemplo, la arquitectura foliar, incluyendo la forma de las láminas, el tipo de tricomas y los patrones de venación se han descrito para no más de tres especies de Rhus s.l. (Hickey y Wolfe, 1975; Terrazas, 1994; Ramírez y Cevallos-Ferriz, 2002; Aguilar-Ortigoza et al., 2004; Martínez y Cevallos-Ferriz, 2005). Hardin y Phillips (1985) reconocen dos tipos de tricomas en cinco especies de Rhus subgénero Rhus, los aciculares con micropapilas estriadas y los glandulares bulbosos, así como cutículas estriadas, mientras que Wilkinson (1979) señala las epidermis estriadas en $R$. thyphina y los estomas menores a $6 \mu \mathrm{m}$ en $R$. copallina. No existe ningún estudio que describa la anatomía foliar y del pecíolo de todas las especies de Rhus s. str. Desde un punto de vista anatómico, la hoja es quizás el órgano más variado de las angiospermas. No obstante, numerosos trabajos han dado mérito al estudio de la anatomía foliar (Metcalfe y Chalk, 1950; Smith y Stern, 1962; Baas y Kool, 1983; Wilkinson, 1983; Bakker y Gerritsen, 1992; Bakker et al., 1992; Metink y Baas, 1992; Dickison y Weitzman, 1996; SandovalZapotitla y Terrazas, 2001; Alves et al., 2002; Aoyama y Sajo, 2003; Fariña et al., 2003) y del pecíolo (Howard, 1979; dos Reis et al., 2004) para apoyar el reconocimiento de grupos taxonómicos supra e infragenéricos. Por ello, nuestro objeto fue describir y comparar los caracteres de la anatomía foliar y del pecíolo de las especies de Rhus s. str. y evaluar si existen atributos anatómicos que apoyen la clasificación infragenérica existente de este género.

\section{Materiales y métodos}

Como parte de un estudio sobre la arquitectura foliar en el género Rhus s. str. se revisaron cerca de 700 ejemplares pertenecientes a 33 especies (la lista completa se puede solicitar a la primera autora). Durante esta revisión se seleccionaron y removieron uno o dos folíolos u hojas, así como sus respectivos pecíolos, de las 33 especies de Rhus s. str. de los herbarios ANSM, ARIZ, DUKE, GH, IEB, NCU, NY, TEX y US (apéndice 1). Desafortunadamente, no fue posible tener material de $R$. chinensis Mill., $R$. potaninii Maxim. y $R$. punjabensis Stewart in Brandis del subgénero Rhus. Las láminas foliares y los pecíolos de 43 individuos fueron rehidratados con hidróxido de sodio al $5 \%$, fijados en formaldehído-ácido acético-alcohol etílico (Johansen, 1940) y procesados con la microtecnia convencional de inclusión en parafina. Una vez incluidas, se hicieron cortes transversales y paradermales a un grosor de $15 \mu \mathrm{m}$ con un micrótomo rotatorio; se tiñeron con safranina y verde rápido y se montaron en resina sintética. Los tejidos de las láminas se describen de acuerdo con Wilkinson (1979) y Mentink y Baas (1992), y de los pecíolos de acuerdo con Howard (1979).

\section{Resultados}

\section{Lámina}

- Cutícula. La cutícula adaxial y abaxial es lisa en la mayoría de las especies (figura 2a), pero 12 de ellas tienen cutícula estriada (figura $2 b$, cuadro 1 ). La cutícula fluctúa desde 
$2 \mu \mathrm{m}$ en $R$. trilobata hasta $13 \mu \mathrm{m}$ en $R$. ovata, aunque la mayoría tiene valores entre 3 y $5 \mu \mathrm{m}$ (cuadro 1 ).

- Epidermis. La epidermis adaxial es simple en todas las especies estudiadas, de forma redondeada, ovalada o cuadrada (figura 2a, b), y comúnmente son redondas en la epidermis abaxial (figura 2c). Sin embargo, en la mayoría de las especies se presentan células papilosas en la lámina cerca de la vena media, con excepción de 12 especies de ambos subgéneros (cuadro 1). Las células de la epidermis adaxial miden desde $17.5 \mu \mathrm{m}$ de ancho en $R$. kearneyi hasta $71.7 \mu \mathrm{m}$ en $R$. palmeri, mientras que las células de la epidermis abaxial más pequeñas son desde $12.5 \mu \mathrm{m}$ de ancho en $R$. kearneyi hasta $48.5 \mu \mathrm{m}$ en $R$. allophylloides. - Mesófilo. El mesófilo es bifacial en todas las especies. En la mayoría de ellas se presenta una capa de parénquima en empalizada; en 13 especies se encuentran dos capas y

Cuadro 1. Atributos de la anatomía foliar y del pecíolo de las especies de Rhus $s$. str. $\mathrm{A}=$ ausente, $\mathrm{d}=\mathrm{drusa}$, dpr $=$ drusa y prismático, $\mathrm{E}=$ estria$\mathrm{da}, \mathrm{G}=$ fibra gelatinosa, $\mathrm{L}=$ lisa, $\mathrm{P}=$ presente, $\mathrm{pr}$. = cristal prismático, vm = vena media. Ver texto para tipo de haz vascular.

\begin{tabular}{|c|c|c|c|c|c|c|c|c|c|c|c|c|}
\hline \multirow{2}{*}{$\begin{array}{l}\text { Subénero/Sección } \\
\text { Especie }\end{array}$} & \multicolumn{9}{|c|}{ Lámina } & \multicolumn{3}{|c|}{ Pecíolo } \\
\hline & $\begin{array}{l}\text { Grosor } \\
\text { cutícula } \\
(\mu \mathrm{m})\end{array}$ & Cutícula & $\begin{array}{l}\text { Papila } \\
\text { lámina }\end{array}$ & $\begin{array}{c}\text { Número } \\
\text { estratos } \\
\text { empalizada }\end{array}$ & $\begin{array}{l}\text { Tipo } \\
\text { cristal }\end{array}$ & $\begin{array}{c}\text { Papilla } \\
\text { vm }\end{array}$ & $\begin{array}{l}\text { Tipo haz } \\
\text { vascular } \\
\text { vm }\end{array}$ & $\begin{array}{c}\text { Fibras } \\
\text { vm }\end{array}$ & $\begin{array}{l}\text { Número } \\
\text { canales }\end{array}$ & Papilas & $\begin{array}{l}\text { Número } \\
\text { estratos }\end{array}$ & $\begin{array}{l}\text { Fibras } \\
\text { xilema }\end{array}$ \\
\hline
\end{tabular}

\section{Rhus subg. Rhus}

R. copallina

R. coriaria

R. glabra

$R$. lanceolata

R. michauxii

$R$. sandwicensis

$R$. typhina

Rhus subg. Lobadium

sección Lobadium

$R$. allophylloides

$R$. aromatica

R. microphylla

R. schmidelioides

R. trilobata

3.5

4.1

3.0

3.5

3.3

3.2

3.9 $\mathrm{vm}$

\section{sección Terebinthifolia}

R. barclayi

R. costaricensis

R. hartmani

R. jaliscana

R. palmeri

R. rubifolia

$R$. terebinthifolia

\section{sección Composita}

$R$. andrieuxii

$R$. choriophylla

R. lentii

R. nelsonii

$R$. oaxacana

R. pachyrrhachis

$R$. schiedeana

$R$. virens

sección Intermedia

$R$. chondroloma

sección Styphonia

$R$. integrifolia

R. kearneyi

R. muelleri

R. ovata

R. standleyi

8.6
7.4
6.8
13.1
7.2

7.2

$\begin{array}{ll}\text { E } & \text { A } \\ \text { E } & \text { A } \\ \text { E } & \text { A } \\ \text { E } & \text { A } \\ \text { E } & \text { A } \\ \text { E } & \text { A } \\ \text { E } & \text { A }\end{array}$

$E$
$E$
$E$
$E$
$E$

A
A
A
A
A

$\begin{array}{ll}d & A \\ d & A \\ d & A \\ d & A \\ d & \text { A }\end{array}$

A
A
A
A
A

$\begin{array}{cc}\text { I } & \text { A } \\ \text { III } & \text { A } \\ \text { III } & \text { A } \\ \text { III } & \text { A } \\ \text { III } & \text { A }\end{array}$

$2-3$
$1-2$
1
$1-2$
1

$\begin{array}{lll}\text { A } & 10 & \text { A } \\ \text { A } & 10 & \text { A } \\ \text { A } & 10 & \text { A } \\ \text { A } & 10 & \text { A } \\ \text { A } & 10 & \text { A } \\ \text { A } & 10 & \text { A } \\ \text { A } & 10 & \text { A }\end{array}$
A A A

$\begin{array}{ll}\mathrm{P} & 1 \\ \mathrm{P} & 1 \\ \mathrm{P} & 1 \\ \mathrm{P} & 1 \\ \mathrm{P} & 1 \\ \mathrm{P} & 1 \\ \mathrm{P} & 1\end{array}$

$$
\text { d }
$$$$
\text { d }
$$$$
\text { P }
$$

$\begin{array}{lll}\text { III } & \text { A } & 1 \\ \text { III } & \text { A } & 1 \\ \text { III } & \text { A } & 3 \\ \text { III } & \text { A } & 1 \\ \text { III } & \text { A } & 1 \\ \text { III } & \text { A } & 3 \\ \text { III } & \text { A } & 1\end{array}$

$\begin{array}{lll}A & <10 & \text { A } \\ \text { A } & <10 & \text { A } \\ \text { A } & <10 & \text { A } \\ \text { A } & <10 & \text { A } \\ \text { A } & <10 & \text { A }\end{array}$

$<10$

pr

P

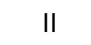

$<10$

P

$\begin{array}{ll}d p r & P \\ d p r & P \\ d p r & P \\ d p r & P \\ d p r & P\end{array}$

$\begin{array}{ll}\text { IV } & P \\ \text { IV } & P \\ \text { IV } & P \\ \text { IV } & P \\ \text { IV } & P\end{array}$

$\begin{array}{ll}\mathrm{P} & 4 \\ \mathrm{P} & 4 \\ \mathrm{P} & 4 \\ \mathrm{P} & 5 \\ \mathrm{P} & i ?\end{array}$




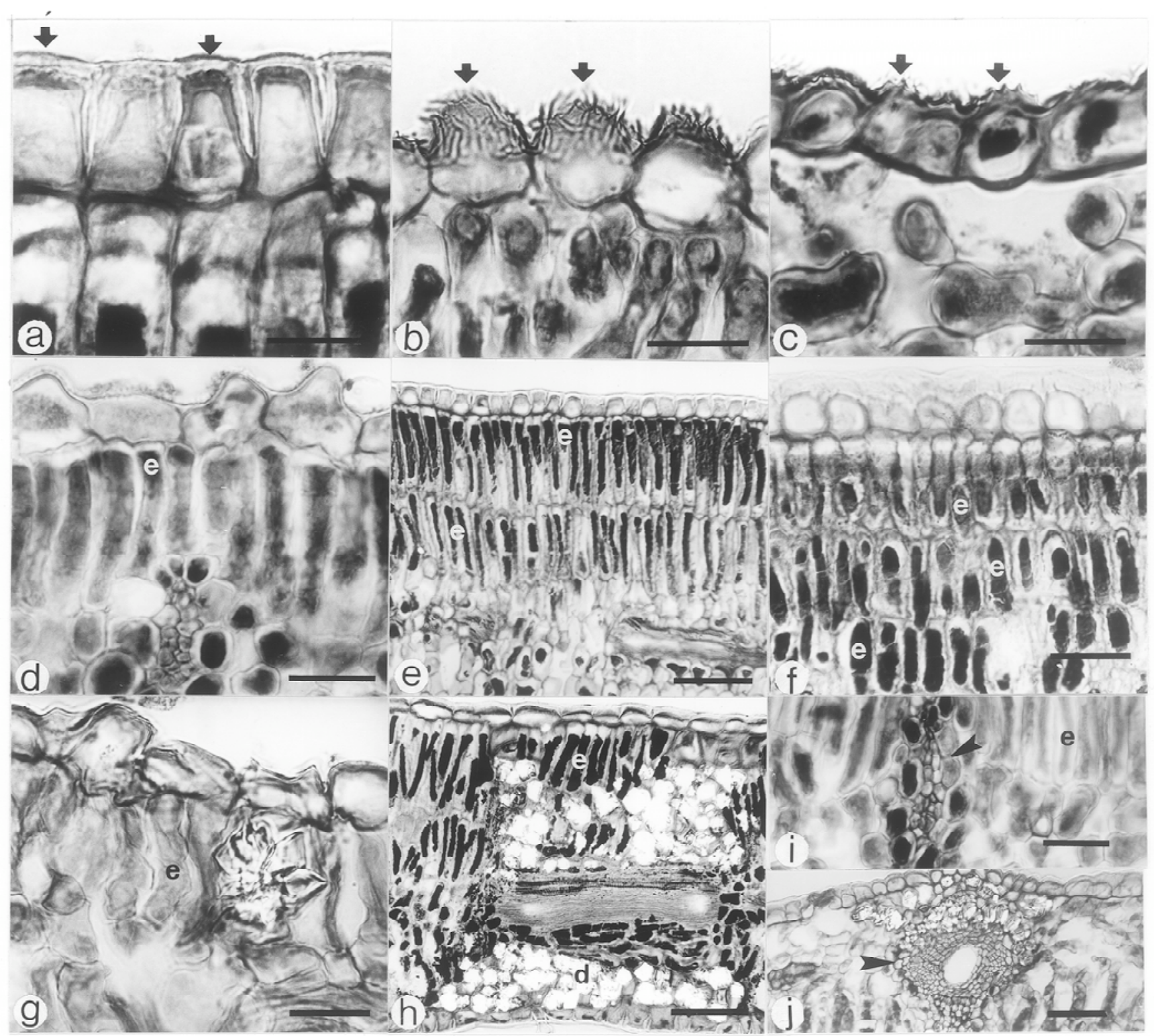

Figura 2. Anatomía foliar. a. Rhus integrifolia (D. Keil 13688, TEX), epidermis adaxial con cutícula lisa (flechas). b. $R$. trilobata (P. Tenorio y C. Romero 6491, IEB), epidermis adaxial con cutícula estriada (flechas). c. $R$. trilobata (P. Tenorio y C. Romero 6491, IEB), epidermis abaxial con cutícula estriada (flecha). d. $R$. terebinthifolia (R. Cedillo 1745, IEB), una capa de parénquima en empalizada. e. R. integrifolia (D. Keil 13688, TEX), dos capas de parénquima en empalizada. f. $R$. ovata (H.S. Gentry 8979, US), tres capas de parénquima en empalizada. g. R. microphylla (Wornock s.n., TEX), drusas en parénquima en empalizada. h. R. muelleri (J.M. Poole 2476, IEB), cristales prismáticos y drusas en mesófilo (se observan blancos por luz polarizada). i. $R$. trilobata (P. Tenorio y C. Romero 6491, IEB), haz vascular sin canal (flecha). j. $R$. andrieuxii (Barkley s.n., NY), haz vascular con canal y fibras (flecha).

Escalas: $\mathbf{a}, \mathbf{b}, \mathbf{d}, \mathbf{g}, \mathbf{i}=20 \mu \mathrm{m}, \mathbf{c}=30 \mu \mathrm{m}, \mathbf{e}=100 \mu \mathrm{m}, \mathrm{f}, \mathrm{h}, \mathrm{j}=50 \mu \mathrm{m}$. La letra e muestra el parénquima en empalizada. 
en $R$. ovata tres capas (figura 2d-f; cuadro 1). Cuando es más de una, existen diferencias en el tamaño de las células que conforman el parénquima en empalizada. Por ejemplo, las células son igualmente largas en $R$. integrifo lia, $R$. kearneyi, $R$. lentii, $R$. muelleri y $R$. standleyi, mientras que las células de la empalizada internas son más cortas en $R$. andrieuxii, $R$. chondroloma, $R$. choriophylla, $R$. nelsonii, $R$. oaxacana, $R$. ovata, $R$. pachyrrachis, $R$. schiedeana y $R$. virens. El parénquima esponjoso tiene entre tres y cuatro capas, excepto en $R$. integrifolia, $R$. keameyi, $R$. lentii, $R$. muelleri, $R$. ovata y $R$. standleyi, en las que se presentan de cinco a siete capas. Las células del esponjoso tienen forma irregular y son de diferentes tamaños, con espacios intercelulares amplios, excepto en seis especies ( $R$. integrifolia, $R$. kearneyi, $R$. lentii, $R$. muel leri, $R$. ovata y $R$. standleyi). En el mesófilo es común la presencia de drusas en la mayoría de las especies (figura 2g); la combinación de cristales prismáticos y drusas es exclusiva de cinco especies, y únicamente se observan cristales prismáticos en ocho especies (cuadro 1). La distribución de ambos tipos de cristales es predominantemente en el parénquima esponjoso, pero también se presentan en el parénquima en empalizada (figura $2 \mathrm{~g}$ ).

- Tejido vascular. En todos los taxones el tejido vascular en la lámina se dispone en haces colaterales. Los haces más pequeños tienen floema escaso, pero difieren en la abundancia del xilema, la presencia de canales y de esclerén- quima. Por ejemplo, sin importar el tamaño de los haces, hay de dos a tres elementos de vaso en xilema, y los canales y esclerénquima están ausentes en once especies pertenecientes a los dos subgéneros (figura $2 \mathrm{i}$ ); en siete especies ( $R$. barclayi, $R$. costaricensis, $R$. hartmanii, $R$. jaliscana, $R$. palmeri, $R$. rubifolia y $R$. terebinthifolia) todos los haces presentan de 2 a 4 elementos de vaso en el xilema, únicamente en los de mayor tamaño hay un canal rodeado por una vaina de células de colénquima, y en el resto de los haces no hay ni canales, ni esclerénquima y colénquima. En el resto de los taxones se presentan de 3 a 15 elementos de vaso en el xilema de todos los haces y sólo en los de mayor tamaño hay un canal y esclerénquima (figura $2 \mathrm{j}$ ).

- Vena media. La anatomía de la vena media difiere entre los taxones examinados (figuras 3, 4a-e). La cutícula en esta región es bastante similar a la zona laminar, pero las células epidérmicas de ambas caras son más pequeñas en todas las especies (figura 4a) y papilosas en 18 especies (cuadro 1). Subepidérmicamente se encuentran entre 2 y 5 capas de colénquima lagunar en ambas caras (figura 4a), y entre el colénquima y el tejido vascular se observan células de parénquima que pueden tener su lumen ocluido por inclusiones minerales (cristales prismáticos, drusas) o taninos. El tejido vascular de la vena media está dispuesto en uno o dos haces colaterales, cuyo arreglo permitió reconocer cuatro tipos (figura 3). El tipo I tiene
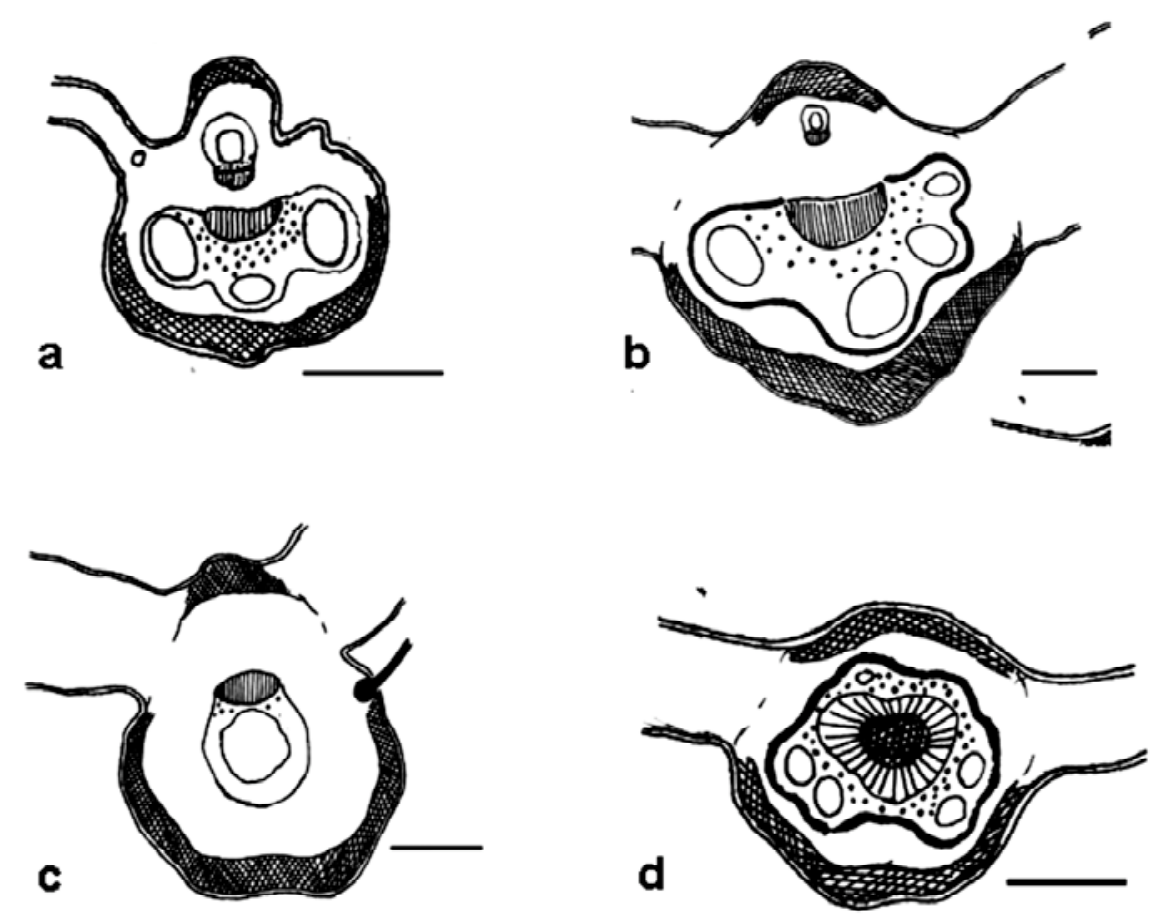

Figura 3. Diagramas de la vena media: forma y tipos del sistema vascular. a. Tipo I, R. typhina (G.M. Baker 555, TEX). b. Tipo II, R. schiedeana (M. Chávez 112, IEB). c. Tipo III, R. jaliscana (M. Huerta y S. Guerrero 256, IBUG). d. Tipo IV, R. ovata (H.S. Gentry 8979, US). Escalas: $\mathbf{a}, \mathbf{b}, \mathbf{d}=50 \mu \mathrm{m}, \mathbf{c}=10 \mu \mathrm{m}$. Clave de símbolos: negro $=$ esclerénquima, puntado $=$ floema, líneas paralelas $=$ xilema, entrecruzado $=$ colénquima, blanco $=$ parénquima, círculos $=$ canales. 


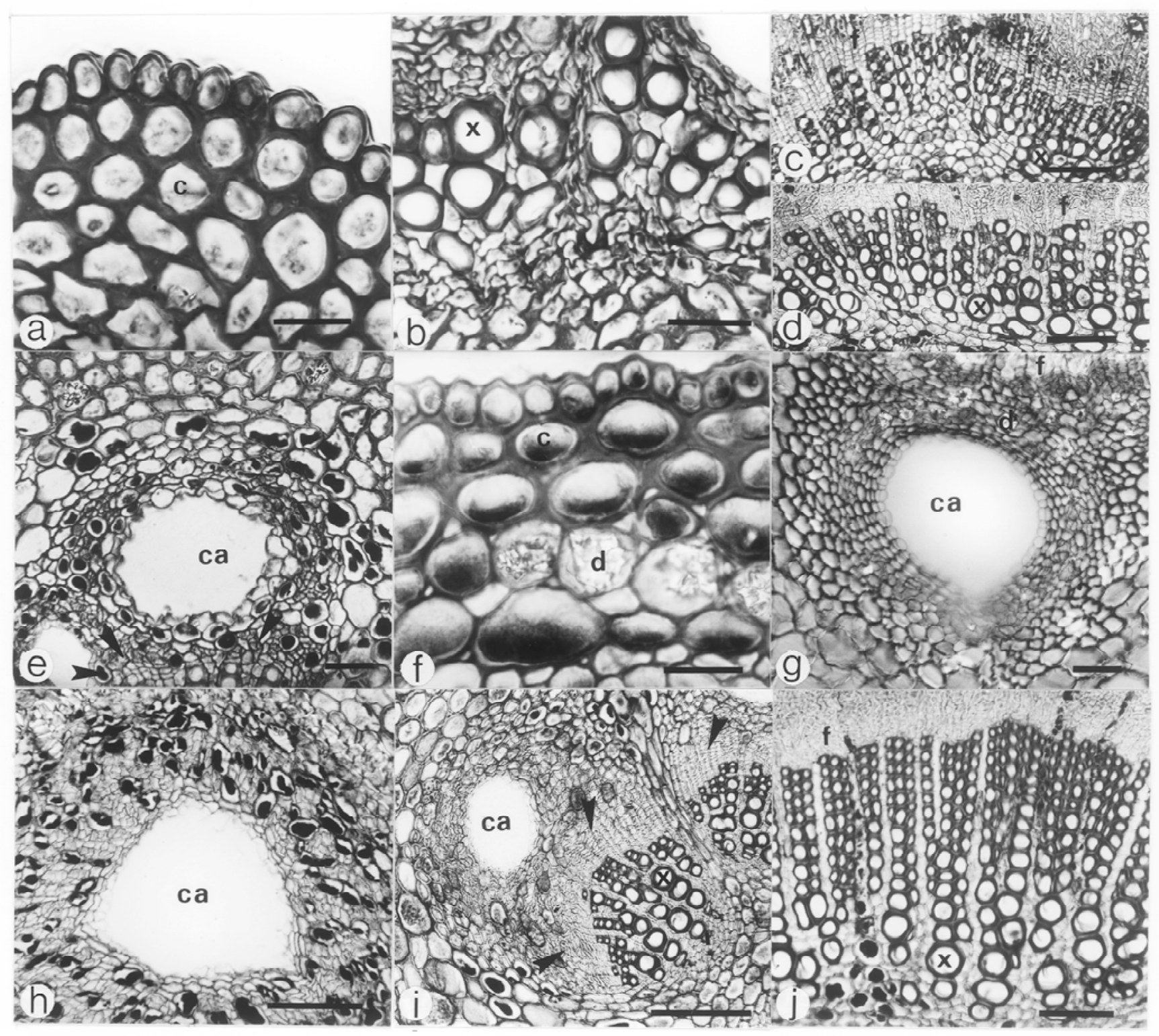

Figura 4. Anatomía foliar y del pecíolo. a-e. Vena media. a. $R$. jaliscana (M. Huerta y $S$. Guerrero 256, IBUG). Colénquima. b. $R$. jalis cana (M. Huerta y S. Guerrero 256, IBUG). Xilema escaso. c. $R$. choriophylla (B. Hinton 16514, US). Xilema abundante. d. $R$. muelleri (J.M. Poole 2476, IEB). Xilema abundante. e. R. trilobata (P. Tenorio y C. Romero 6491, IEB). Canal de la vena media (flecha = floema). f-j. Pecíolo. f. $R$. microphylla (Wornock s.n., TEX). Epidermis y córtex. g. R. typhina (G.M. Baker 555, TEX). Canal asociado al floema. h. $R$. schiedeana (M. Chávez 112, IEB). Canal asociado al floema. i. R. kearneyi (R. Moran 18308, US). Haz vascular rodeado de fibras (flechas). j. R. virens (C.L. Lundell 12477, US). Xilema abundante con fibras.

Eacalas: $\mathbf{a}, \mathbf{f}=20 \mu \mathrm{m}, \mathbf{b}, \mathbf{g}, \mathbf{j}=50 \mu \mathrm{m}, \mathbf{c}-\mathbf{e}, \mathbf{h}=100 \mu \mathrm{m}, \mathbf{i}=200 \mu \mathrm{m} . \mathrm{ca}=$ canal, $\mathrm{c}=$ colénquima, $\mathrm{d}=\mathrm{drusa}, \mathrm{f}=\mathrm{floema}, \mathrm{x}=\mathrm{xilema}$. 
arco simple, abierto, con un haz vascular adicional en la región adaxial y sin fibras que rodean al haz (figura 3a), y se presenta en ocho especies (cuadro 1). El tipo II corresponde a un arco simple, abierto, con un haz vascular adicional en la región adaxial, pero con fibras rodeando el haz (figura 3b); ocho especies tienen este tipo. El tipo III se caracteriza por un arco simple, abierto, sin haz vascular adicional y sin fibras que rodean al haz (figura $3 \mathrm{c}$ ), $\mathrm{y}$ se presenta en 11 especies (cuadro 1). Por último, el tipo IV corresponde a un arco cerrado, con fibras que rodean al haz (figura 3d), y fue encontrado en seis especies (cuadro 1). El xilema presenta los elementos de vaso en hileras radiales, las cuales varían en número de 5 a 20 en la mayoría de las especies (figura $4 \mathrm{~b}$ ), aunque llegan a ser hasta más de 30 hileras en $R$. choriophylla, $R$. muelleri, $R$. oaxacana, $R$. ovata, $R$. schiedeana y $R$. virens (figuras $4 \mathrm{c}$, d), así como fibras y parénquima radial. El floema está constituido por elementos de tubo criboso, células acompañantes y parénquima; en todas las especies estudiadas los canales se disponen sobre el floema y pueden o no presentar vaina del canal (figura 4e). Resalta que $R$. bar clayi, $R$. costaricensis, $R$. jaliscana, $R$. palmeri y $R$. tere binthifolia presentan exclusivamente un canal, mientras que las especies $R$. copallina, $R$. coriaria, $R$. glabra, $R$. michauxii, $R$. sandwicensis y $R$. typhina comúnmente tienen cinco canales, aunque es rara la presencia de cuatro sólo en $R$. copallina.

Pecíolo. El pecíolo, visto en corte transversal, es circular en la mayoría de las especies, oval en $R$. coriaria y $R$. ovata, circular alado en $R$. microphylla, y triangular en $R$. andrieuxii, $R$. costaricensis, $R$. lanceolata, $R$. integrifolia, $R$. jaliscana, $R$. sandwicensis, $R$. standleyi, $R$. kearneyi y $R$. terebinthifolia (figura 5). Los pecíolos más grandes, con una área de $4.63 \mathrm{~mm}$ a $19.02 \mathrm{~mm}$, se encuentran en $R$. glabra y $R$. typhina, y los pecíolos más pequeños, con área entre $0.23 \mathrm{~mm}$ y $1.07 \mathrm{~mm}$, se presentan en $R$. microphylla y $R$. aromatica, respectivamente.

- Epidermis. La epidermis es simple, de células ovadas o redondas en la mayoría de los taxones (figura 4f), pero papilosas en 13 especies (cuadro 1). En todas las especies los tricomas y las glándulas son semejantes a los descritos para la lámina. Sólo se observó peridermis en $R$. costaricensis, $R$. integrifolia, $R$. hartmanii, $R$. jaliscana, $R$. palmeri, $R$. rubifolia, $R$. sandwicensis y $R$. terebinthi folia.

- Córtex. Subepidérmicamente, en la mayoría de las especies se encuentran de 3 a 9 capas de colénquima lagunar, excepto en las especies del subgénero Rhus (cuadro 1 ), en las que se observa ron más de diez capas, seguidas de 3 a 5 capas de parénquima. Los taxones que presentan uno o dos tipos de cristales (drusas / prismáticos / ambos) coincide con lo descrito para la lámina (figura 4f).

- Tejido vascular. El tejido vascular en todas las especies se dispone en un cilindro discontinuo o cerrado (figura 5). A pesar de formar un cilindro continuo, el número de haces vasculares varía entre 3 y 7 en la mayoría de las especies, y sólo en $R$. copallina, $R$. coriaria, $R$. glabra, $R$. lanceo lata, $R$. michauxii, $R$. sandwicensis y $R$. typhina el número de haces es mayor o igual a siete. En cada haz vascular hay un canal asociado al floema con esclerénquima que se limita al haz o que une a los haces. En este canal, además de las células epiteliales, puede presentarse una vaina del canal con 2 a 7 capas de células compactas, ocasionalmente con contenidos obscuros (figuras 4e, g-i). En el xilema de estos haces únicamente se presentan elementos de vaso y parénquima en 12 especies, mientras que en 14 también hay fibras (figuras $4 \mathrm{i}, \mathrm{j}$, cuadro 1) y sólo en siete especies se observan fibras gelatinosas $(R$. barclayi, $R$. costaricensis, $R$. hartmanii, $R$. jaliscana, $R$. palmeri, $R$. rubifolia y $R$. terebinthifolia). La médula es parenquimatosa con drusas y contenidos obscuros abundantes en todos los taxones, y destaca que en $R$. copalli $n a, R$. coriaria, $R$. glabra, $R$. lanceolata, $R$. michauxii, $R$. sandwicensis y $R$. typhina es amplia.

\section{Discusión}

El estudio aquí presentado revela la existencia de caracteres comunes a todas las especies de Rhus s. str., como son la presencia de epidermis simple con tricomas y glándulas y de canales asociados al floema. La presencia de glándul a s y canales son atributos que se comparten con otros taxones de Anacardiaceae y Burseraceae (Metcalfe y Chalk, 1950; Terrazas, 1994; Judd et al., 2002). Sin embargo, hay características de la epidermis, del mesófilo y del tejido vascular que son importantes para diferenciar los grupos infragenéricos (cuadro 2) propuestos por Barkley (1937) y Young (1978). Las especies estudiadas del subgénero Rhus (R. copallina, $R$. coriaria, $R$. glabra, $R$. lanceolata, $R$. michauxii, $R$. sandwicensis y $R$. typhina) se distinguen por presentar un sistema vascular tipo I en la vena media, caracterizado por cinco canales, a la vez que en el pecíolo hay un número superior a diez capas de colénquima, más de siete haces vasculares y una médula amplia. Los miembros del subgénero Rhus comparten con casi todas las especies del subgénero Lobadium secciones Lobadium y Terebinthifolia, una cutícula delgada $(<6 \mu \mathrm{m})$, células de la epidermis no papilosas, una capa de parénquima en empalizada, drusas en mesófilo y la ausencia de fibras en el xilema secundario de la vena media. Sin embargo, los taxones de la sección Terebinthifolia ( $R$. barclayi, $R$. costaricensis, $R$. hartmanii, $R$. jaliscana, $R$. palmeri, $R$. rubifolia y $R$. terebinthifolia) se distinguen por tener de 2 a 4 elementos de vaso en xilema, una vaina colenquimatosa que rodea a los haces vasculares de la lámina, y por presentar fibras gelatinosas en el xilema de la vena media y el pecíolo, así como peridermis en este último. Ningún carácter resultó ser 


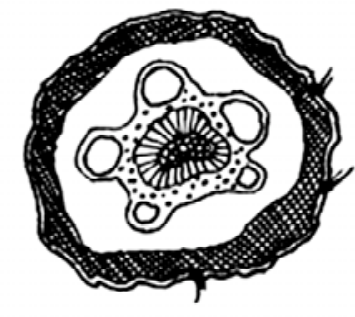

a

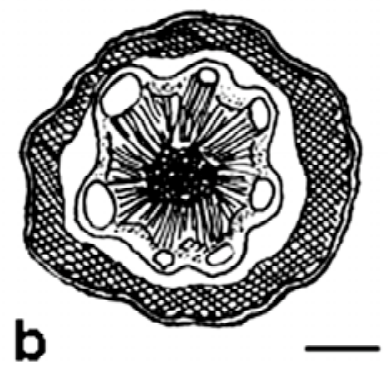

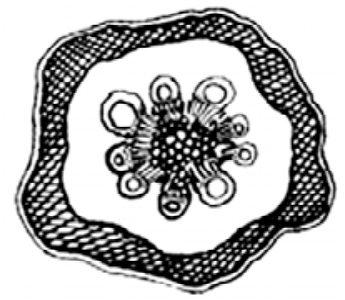

C
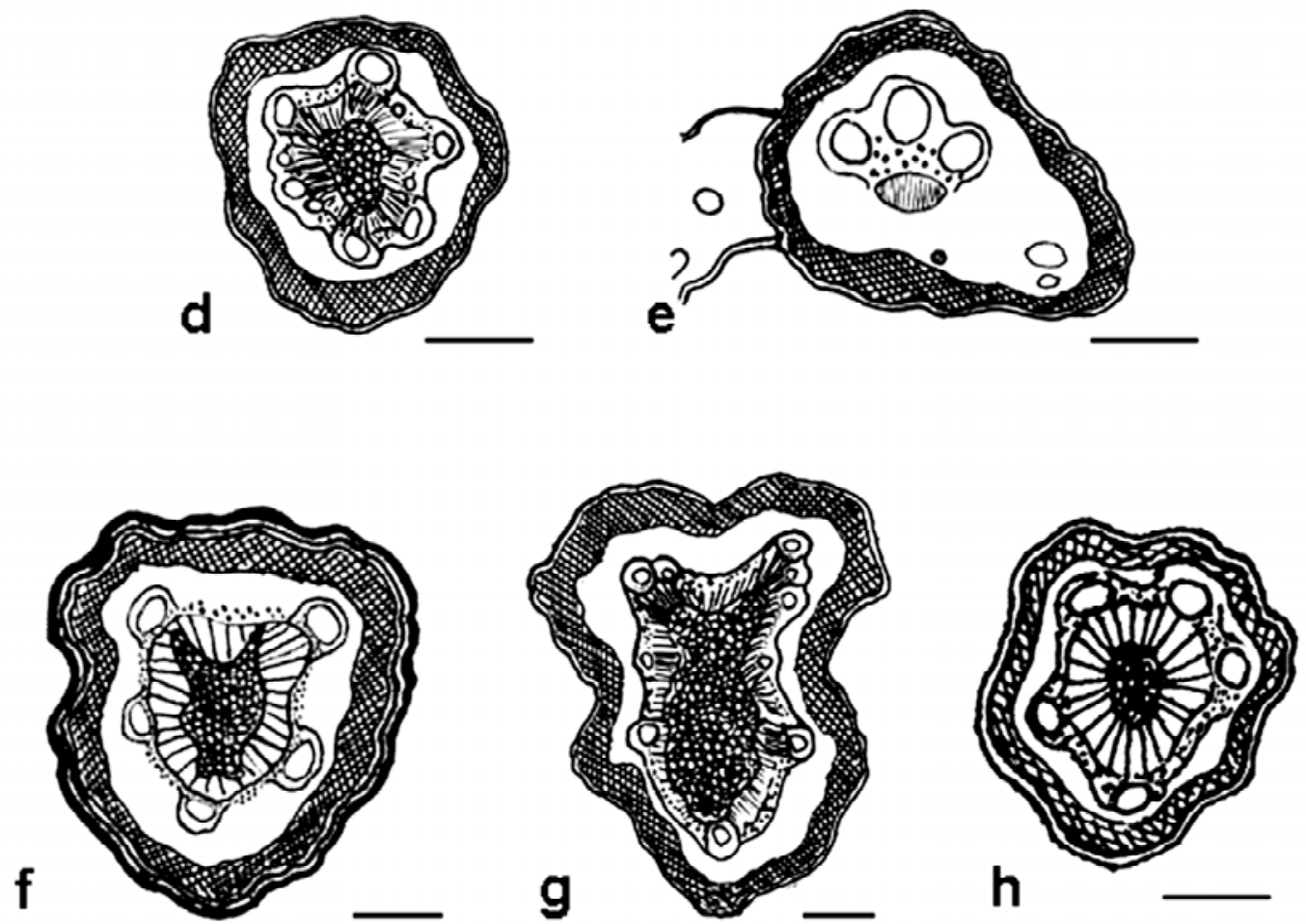

Figura 5. Diagrama de la forma del pecíolo. a-c. Circular. a. $R$. trilobata (P. Tenorio y C. Romero 6491, IEB). b. R. glabra (C.M. Rowell 4175, TEX). c. $R$. chondroloma (F. McCarten 2976, US). d. Oval, $R$. schiedeana (M. Chávez 112, IEB ). e. Circular alado, R. micro phylla (H. Wornock s.n., TEX). f-h. Triangular. f. R. costaricensis (Tonduz 9823, US). g. R. sandwicensis (O. Deneger 9527, TEX). h. $R$. terebinthifolia (R. Cedillo 1745, IEB).

Escalas: a-d, $\mathbf{f}=50 \mu \mathrm{m}, \mathbf{e}=20 \mu \mathrm{m}, \mathbf{g}=100 \mu \mathrm{m}, \mathbf{h}=25 \mu \mathrm{m}$. Clave de símbolos: negro = esclerénquima, puntado = floema, líneas paralelas $=$ xilema, entrecruzado $=$ colénquima, blanco $=$ parénquima, círculos $=$ canales.

exclusivo ni en la lámina ni en el pecíolo de los taxones de la sección Lobadium ( $R$. allophylloides, $R$. aromatica, $R$. microphylla, $R$. schmidelioides y $R$. trilobata). Los taxones de ambas secciones, Lobadium y Terebinthifolia, comparten un sistema vascular tipo III en la vena media.

Los taxones de la sección Styphonia se caracterizan por tener una cutícula gruesa $(>6 \mu \mathrm{m})$, células epidérmicas papilosas en la lámina y vena media, dos capas de parénquima en empalizada y xilema de la vena media y pecíolo con fibras. A pesar de compartir los atributos mencionados, los taxones de cada subsección de la sección Styphonia pueden ser distinguidos por la combinación de varios atributos de la lámina. Por ejemplo, las especies de la subsección Styphonia ( $R$. integrifolia, $R$. keameyi, $R$. lentii, $R$. muelleri, $R$. ovata y $R$. standleyi) se separan por el tamaño similar de las dos capas del parénquima en empalizada, la presencia de cristales prismáticos y drusas en mesófilo de lámina y vena media, así como por el sistema vascular de tipo IV en la vena media. En esta subsección, $R$. ovata se distingue por tener tres capas de parénquima en empalizada. Las especies de las subsecciones Composita (R. andrieuxii, $R$. choriophylla, R. nelsonii, R. oaxacana, R. pachyrrhachis, 
Cuadro 2. Características de la anatomía foliar y del pecíolo presentes en las categorías infragenéricas de Rhus s. str. Entre paréntesis se encuentra el estado de carácter raro por estar presente en sólo una o dos especies.

\begin{tabular}{|c|c|c|c|c|c|c|}
\hline \multirow{3}{*}{$\begin{array}{l}\text { Subgénero } \\
\text { Sección } \\
\text { Subsección }\end{array}$} & \multirow[t]{3}{*}{ Rhus } & \multicolumn{5}{|c|}{ Lobadium } \\
\hline & & \multirow[t]{2}{*}{ Lobadium } & \multirow[t]{2}{*}{ Terebinthifolia } & \multicolumn{3}{|c|}{ Styphonia } \\
\hline & & & & Styphonia & Composita & Intermedia \\
\hline \multicolumn{7}{|l|}{ Lámina } \\
\hline Espesor cutícula & $<6 \mu \mathrm{m}$ & $<6 \mu \mathrm{m}$ & $<6 \mu \mathrm{m}$ & $>6 \mu \mathrm{m}$ & $>6 \mu \mathrm{m}$ & $>6 \mu \mathrm{m}$ \\
\hline Cutícula & estriada & estriada & lisa & lisa & lisa & lisa \\
\hline Epidermis papilosa & - & - & + & + & + & + \\
\hline $\begin{array}{l}\text { Número de capas de parénquima en } \\
\text { empalizada }\end{array}$ & 1 & 1 & 1 & 2 & $\begin{array}{c}2 \\
\text { desiguales }\end{array}$ & $\begin{array}{c}2 \\
\text { desiguales }\end{array}$ \\
\hline Cristales mesófilo & drusas & drusas & drusas & $\begin{array}{l}\text { drusas, } \\
\text { prismáticos }\end{array}$ & prismáticos & prismáticos \\
\hline \multicolumn{7}{|l|}{ Vena media } \\
\hline Epidermis papilosa & - & - & - & + & + & + \\
\hline Número de canales & 5 & $2-3$ & 1 & $4-5$ & $2-5$ & 2 \\
\hline Xilema con fibras & - & - & - & + & + & + \\
\hline Arreglo vascular & 1 & $\mathrm{III}(\mathrm{I})$ & III & IV & $\mathrm{II}(\mathrm{IV})$ & II \\
\hline \multicolumn{7}{|l|}{ Pecíolo } \\
\hline Cilindro vascular & discontinuo & continuo & $\begin{array}{l}\text { discontinuo / } \\
\text { continuo }\end{array}$ & $\begin{array}{l}\text { discontinuo/ } \\
\text { continuo }\end{array}$ & $\begin{array}{l}\text { discontinuo/ } \\
\text { continuo }\end{array}$ & continuo \\
\hline Número de canales & $>7$ & $<7$ & $<7$ & $<7$ & $<7$ & $<7$ \\
\hline Xilema con fibras & - & - & - & + & + & + \\
\hline Xilema con fibras gelatinosas & - & - & + & - & - & - \\
\hline
\end{tabular}

$R$. schiedeana y $R$. virens) e Intermedia ( $R$. chondroloma) se reconocen del resto de las especies del género Rhus $s$. $s t r$. por tener dos capas de células en empalizada y la interna más corta, por la presencia de cristales prismáticos en el mesófilo y la vena media, y por un sistema vascular tipo II en la vena media.

Hardin y Phillips (1985) hallaron cutículas estriadas en cinco especies de Rhus subgénero Rhus (R. copallina, $R$. glabra, $R$. lanceolata, $R$. michauxii y $R$. typhina). Nosotras también observamos una cutícula estriada en $R$. sandwicen sis, así como en las cinco especies del subgénero Lobadium sección Lobadium. Sin embargo, las diferencias encontradas en la posición, la abundancia y el grosor de la estriación deberán estudiarse con microscopio electrónico de barrido con la finalidad de confirmar si los taxones de la sección Lobadium tienen estriaciones más gruesas.

La presencia de dos capas de parénquima ha sido reconocida en otros géneros de la familia como Gluta (Wilkinson, 1983) y Malosma (Andrés-Hernández, datos sin publicar), y nuestras observaciones mostraron que es un atributo distintivo de las especies de la sección Styphonia. Dickison (2000) atribuye la presencia de más de una capa de parénquima en empalizada a la exposición continua al sol de la hoja. Sin embargo, Kornelius (1964) opina que el número de capas en empalizada puede estar fijado genéticamente. Nosotras coincidimos en que el número de capas del parénquima en empalizada presente en las especies de Rhus s. str. es un atributo fijado genéticamente. Por ejemplo, especies que viven en simpatría como Rhus aromatica (sección Lobadium) y Rhus standleyi (sección Styphonia) mantienen sus diferencias en el número de capas del parénquima en empalizada, a pesar de estar expuestas a condiciones semejantes de exposición al sol. Esta aseveración hace interesante el diseño de un estudio empírico donde se ponga a prueba esta hipótesis.

La disposición y la abundancia del esclerénquima en la lámina han sido consideradas rasgos importantes para apoyar la distinción de grupos taxonómicos (Smith y Stern, 1962; Wilkinson, 1983; Dickison, 1989, 1996; Fariña et al., 2003). Los géneros Guioa, Cupaniopsis y Harpulla de las Sapindales presentan esclerénquima asociado al cilindro vascular (Buijsen, 1995). En el caso específico del género Rhus s. str., el esclerénquima asociado al tejido vascular se restringe a la vena media y en pocas especies a las venas de menor orden como secundarias y terciarias (AndrésHernández y Terrazas, en revisión). Para la vena media, este estudio permitió reconocer cuatro tipos que coinciden con la clasificación infragenérica. Los tipos I (subgénero Rhus) y III (secciones Lobadium y Terebinthifolia) carecen de esclerénquima o presentan colénquima y fibras exclusivamente gelatinosas, mientras que los tipos II y IV, los cuales presentan fibras tanto en el floema como en el xile- 
ma, se hallan en los taxones de la sección Styphonia. Nosotras atribuimos las diferencias en esclerificación a la persistencia de la hoja en las especies de Rhus s. str. En la sección Styphonia, las especies tienen hojas simples o imparipinnado compuestas, persistentes por más de un año, con una longitud que fluctúa entre $3.4 \mathrm{~cm}$ en $R$. kearneyi y $25 \mathrm{~cm}$ en $R$. oaxacana, y todas ellas se distinguen por la presencia de esclerénquima asociado a la vena media en xilema o sobre floema, dos capas de parénquima en empalizada y cutículas lisas con un espesor mayor que $6 \mu \mathrm{m}$. Los taxones de Styphonia se distribuyen preferentemente en matorrales xérofilos o en las zonas de transición entre estos matorrales y el bosque templado, donde las condiciones de aridez y exposición a la luz seguramente han favorecido la presencia de estos atributos como posibles novedades evolutivas a las nuevas condiciones ambientales.

Varios autores han señalado que la anatomía del pecíolo es útil en la sistemática de diversos grupos (Metcalfe y Chalk, 1950; Howard, 1979; Engel, 1992; dos Reis et al., 2004; Souza et al., 2004). La anatomía del pecíolo, como ya se mencionó, apoya la circunscripción de las categorías infragenéricas de Rhus s. str. Sólo los pecíolos de Rhus subg. Rhus presentan una médula amplia, abundante colénquima y ausencia de esclerénquima. Seguramente estos atributos le confieren flexibilidad y tienen un menor costo energético, ya que sus hojas imparipinnado compuestas, además de ser grandes (59 cm en longitud, $R$. typhina), son caducas. Como señala Givnish (1978), producir hojas compuestas de vida corta tiene un menor costo energético en comparación con las hojas simples de pecíolos lignificados, y ésta se considera como una de varias estrategias que favorecen el rápido crecimiento vertical en ambientes con estacionalidad marcada. Esta interpretación se da no sólo a los taxones de Rhus subg. Rhus, sino también a los miembros de la sección Lobadium. Engel (1992) interpreta la presencia de esclereidas en el pecíolo de Astragalus como un carácter asociado a la persistencia de las hojas. Esta interpretación también se puede dar a las especies de la sección Styphonia, con hojas persistentes y con fibras rodeando el sistema vascular, como ya se mencionó para la vena media.

Concluimos que los atributos del sistema vascular de la vena media, así como de la epidermis, del mesófilo y del pecíolo, permiten reconocer los subgéneros de Rhus s. str., confirmando las hipótesis propuestas con base en caracteres moleculares (Miller et al., 2001; Yi et al., 2004). Además, las secciones también se reconocen por la combinación única de rasgos anatómicos foliares y del pecíolo (cuadro 2). A pesar de que la posición de la inflorescencia y la variabilidad en el número de foliolos condujeron a Young (1978) a proponer la subsección Intermedia para $R$. chondroloma, nuestros resultados señalan una fuerte similitud en la anatomía foliar entre esta especie y los taxones de la subsección Composita. Estas observaciones apoyan la similitud reportada en los patrones de venación entre ambas subsecciones (Andrés-Hernández y Terrazas, en revisión); por ello sugerimos transferir $R$. chondroloma a la sección Composita.

\section{Agradecimientos}

La primera autora agradece al CONACYT (No. 169599) la beca para realizar estudios de doctorado; a los curadores de los herbarios ANSM, ARIZ, DUKE, GH, IEB, NCU, NY, TEX y US por permitirnos remover material para este estudio y a Héctor Hernández por el trabajo de cuarto obscuro.

\section{Literatura citada}

Aguilar-Ortigoza C., Sosa V. y Ángeles G. 2004. Phylogenetic relationships of three genera in Anacardiaceae: Bonetiella, Pseudosmodingium and Smodingium. Brittonia 56:169-184.

Alves M.V., Estelita M.E.M., Wanderley M.G.L y Thomas W.W. 2002. Aplicações taxonômicas da anatomia foliar das especies brasileiras de Hypolytrum Rich (Cyperaceae). Revista Brasileira de Botânica 25:1-9.

Andrés-Hernández A.R. y Terrazas T. en revisión. Leaf architecture of Rhus s. str. (Anacardiaceae). Plant Research Journal.

Aoyama E.M. y Sajo M.G. 2003. Estructura foliar de Aechmea Ruiz \& Pav. Subgênero Lamprococcus (Beer) Baker e espécies relacionadas (Bromeliaceae). Revista Brasileira de Botânica 26:461-473.

Baas P. y Kool R. 1983. Comparative leaf anatomy of Heisteria (Olacaceae). Blumea 28:367-388.

Bakker M.E. y Gerritsen A.F. 1992. Oil and mucilage cells in Annona (Annonaceae) and their systematic significance. Blumea 36:411-438.

Bakker M.E., Gerritsen A.F. y van der Schaaf P.J. 1992. Leaf anatomy of Cinnamomum Schaeffer (Lauraceae) with special reference to oil and mucilage cells. Blumea 37:1-30.

Barkley F.A. 1937. A monographic study of Rhus and its immediate allies in North and Central America, including the West Indies. Annals of the Missouri Botanical Garden 24:256-500.

Buijsen J.R.M. 1995. Leaf anatomy of Harpullia, Majidea, and Conchopetalum (Sapindaceae). Blumea 40:345-361.

Dickison W.C. 1989. Stem and leaf anatomy of the Alseuosmiaceae. Aliso 3:567-578.

Dickison W.C. 1996. Stem and leaf anatomy of Saruma henryi Oliv., in cluding observations on raylessness in the Aristolochiaceae. Bulletin of the Torrey Botanical Club 4:261-267.

Dickison W.C. 2000. Integrative Plant Anatomy. Academic Press, San Diego.

Dickison W.C. y Weitzman A.L. 1996. Comparative anatomy of the young stem, node, and leaf of Bonnetiaceae, including observations on a foliar endodermis. American Journal of Botany 83:405-418.

Engel T. 1992. Petiolar anatomy of North American Astragalus species (Fabaceae) with persistent petioles. Aliso 2:339-345.

Fariña A., Arrieche D., Boada-Sucre A. y Velázquez D. 2003. Anatomía comparada de la lámina foliar de las especies de Heliotropium L. (Boraginaceae) presentes en Venezuela. Interciencia 28:68-74. 
Givnish T.J. 1978. On the adaptive significance of compound leaves, with particular reference to tropical trees. En: Tomlinson P.B. y Zimmermann M.H. Eds. Tropical Trees as Living Systems, pp. 351-380, Cambridge University Press, Cambridge.

Hardin J.W. y Phillips L.L. 1985. Atlas of foliar surface features in woody plants, VII. Rhus subg. Rhus (Anacardiaceae) of North America. Bulletin of the Torrey Botanical Club 112:1-10.

Hickey L.J. y Wolfe J.A. 1975. The bases of angiosperm phylogeny: vegetative morphology. Annals of the Missouri Botanical Garden 62:538-589.

Howard R.A. 1979. The petiole. En: Metcalfe C.R. y Chalk L. Eds. Anatomy of the Dicotyledon. Vol. I, 2a ed., pp. 88-96, Clarendon Press, Oxford.

Johansen D.A. 1940. Plant Microtechnique. McGraw-Hill, Nueva York.

Judd W.S., Campbell C.S., Kellogg E.A., Stevens P.F. y Donoghue M.J. 2002. Plant Systematics, a Phylogenetic Approach. 2a ed., Sinauer, Sunderland.

Kornelius L. 1964. Evolutionary studies in the Ericaceae. II. Leaf anatomy as a phylogenetic index in the Andromedeae. Botanical Gazette 125:178-186.

Martínez-Millán M. y Cevallos-Ferriz S.R.S. 2005. Arquitectura foliar de Anacardiaceae. Revista Mexicana de Biodiversidad 76:137-190.

Mentink H. y Baas P. 1992. Leaf anatomy of the Melastomataceae, Memecylaceae and Cryptoniaceae. Blumea 37:189-225.

Metcalfe C.R. y Chalk L. 1950. Anatomy of the Dicotyledons. 1a ed., Clarendon Press, Oxford.

Miller A.J., Young D.A. y Wen J. 2001. Phylogeny and bioge ography of Rhus (Anacardiaceae) based on ITS sequence data. International Journal of Plant Science 162:1401-1407.

Ramírez J.L. y Cevallos-Ferriz S.R.S. 2002. A diverse assemblage of Anacardiaceae from Oligocene sediments, Tepexi de Rodríguez, Puebla, México. American Joumal of Botany 89:535-545.

Reis C. Dos, Proenca S.L. y Sajo M.G. 2004. Vascularização foliar e anatomia do pecíolo de Melastomataceae do cerrado do Estado de São Paulo, Brasil. Acta Botanica Brasilica 18:987999.

Sandoval-Zapotitla E. y Terrazas T. 2001. Leaf anatomy of 16 taxa of the Trichocentrum clade (Orchidaceae:Oncidiinae). Lindleyana 16:81-93.

Smith A. y Stern W. 1962. Leaf anatomy as an aid in the identification of two Fijian plant species. Brittonia 14:237-247.

Souza L.A., Moscheta I.S. y Oliveira J.H.G. 2004. Comparative morphology and anatomy of the leaf and stem of Peperomia dahlstedtii C.DC., Ottania martiana Miq. and Piper diospyri folium Kunth (Piperaceae). Gayana Botanica 61:6-17.

Terrazas T. 1994. Wood anatomy of the Anacardiaceae: ecological and phylogenetic interpretation. Tesis de Doctorado, University of North Carolina, Chapel Hill, 321 pp.

Yi T., Miller A.J. y Wen J. 2004. Phylogenetic and biogeographic diversification of Rhus (Anacardiaceae) in the northern hemisphere. Molecular Phylogenetics and Evolution 33:861-879.

Young D.A. 1975. Systematics of Rhus subgenus Lobadium section Styphonia. Tesis Doctorado, Claremont Graduate School, Claremont, California, $259 \mathrm{pp}$.

Young D.A. 1978. Reevaluation of the sections of Rhus L. subgenus Lobadium (Raf.) T. \& G. (Anacardiaceae). Brittonia 4:411-415.

Wilkinson H.P. 1979. Cuticle. En: Metcalfe C.R. y Chalk L. Eds. Anatomy of the Dicotyledons. Vol. 1, 2a ed., pp. 140-155, Clarendon Press, Oxford.

Wilkinson H.P. 1983. Leaf anatomy of Gluta (L.) Ding Hou (Anacardiaceae). Botanical Journal of the Linnean Society 86:375-403.

Fecha de recepción: 17 de octubre de 2005

Versión corregida: 28 de abril de 2006

Aceptado: 2 de mayo de 2006 


\section{Agustina Rosa Andrés-HernándeZ y TERESA TERraZAS}

Apéndice 1. Especies de Rhus s. str. arregladas en orden alfabético por subgénero, sección y subsección con los especímenes utilizados para el estudio anatómico foliar y del pecíolo con colector y herbario de acuerdo con Holmgren et al. (1981).

Rhus subgénero Rhus: R. copallina L. (F.R. Waller et al. 2806, TEX; R. Bochinski s.n., US); R. coriaria L. (D. Goldman 1080, NY); R. glabra L. (Chester y M. Rowell 4175, TEX); R. lanceolata (A.Gray) Britton (D.S. Correll 38069, TEX); R. michauxii Sarg. (H.E. Ahles 58816, NCU); R. sandwincensis A.Gray (O. Deneger 9527, TEX; S. Darwin 1201, TEX); R. typhina L. (G.M. Baker 555, TEX; A. Hayden 7161, TEX).

Rhus subgénero Lobadium sección Lobadium: R. allophylloides Standl. (G.B. Hinton 2817; US); R. aromatica Aiton (Gómez Durán s.n., IEB; M. Nee 44041, TEX); R. microphylla Engelm. ex A.Gray (H. Wornock s.n., TEX); R. schmidelioides Schltdl. (H. Díaz-Barriga 3516, IEB); R. trilobata Nutt. ex Torr. et A.Gray (P. Tenorio y C. Romero 6491, IEB). Sección Terebinthifolia: $R$. bar clayi (HemsI.) StandI. (R. Ornelas 1604, 1614, IBUG); R. costaricensis L.Riley (W.T.Gillis 9100, TEX; Tonduz 9823, US); R. hart manii F.A.Barkley (L.J. Toolin 310, ARIZ); R. jaliscana Standl. (M. Huerta y S. Guerrero 256, IBUG; C.G. Pringlei s.n., US); R. palmeri Rose (P.C. Standley et al. 1310, GH); R. rubifolia Turcz. (R. Ornelas 1545, ANSM); R. terebinthifolia Schltdl. et Cham. ( $R$. Cedillo 1745, IEB). Sección Styphonia subsección Styphonia: R. integrifolia (Nutt. ex Torr. et A.Gray) Benth. et Hook.f. ex Rothr. (H.M. Pollard s.n., TEX; D. Keil 13688, TEX); R. kearneyi F.A.Barkley (R. Moran 18308, US); R. lentii Kellogg (A. Carter 4431, TEX); R. muelleri Standl. et F.A.Barkley (T.F. Petterson et al. 7164, TEX; M. Poole et al. 2476, IEB); R. ovata S.Watson (H.S. Gentry 8979, US; T. Crovello 270, TEX); R. standleyi F.A.Barkley (M. Luckow 2538, TEX). Sección Styphonia subsección Composita: R. choriophylla Wooton et Standl. (L. Lundell 12477, US; B. Hinton 16514, US); R. oaxacana Loes. (T.B. Croat 46237, DUKE); R. pachyrrhachis Hemsl. (J. Henrickson 19091, TEX); R. schiedeana Schltdl. (M. Chávez 112, IEB); R. virens Lindh. ex A.Gray ); R. choriophylla Wooton et Standl. (B. Hinton 16514, US); R. nelsonii F.A.Barkley (J. Calónico 6548, IEB). Sección Styphonia subsección Intermedia: R. chondroloma Standl. (F. McCarten 2976, US). 